\title{
Role of Oxidative Stress, Adiponectin and Endoglin in the Pathophysiology of Erectile Dysfunction in Diabetic and Non-Diabetic Men
}

\author{
B. TREBATICKÝ ${ }^{1 *}$, I. ŽITŇANOVÁ ${ }^{2} *$ M. DVOŘÁKOVÁ ${ }^{2}$, Z. ORSZÁGHOVÁ $^{2}$, \\ Z. PADUCHOVÁ ${ }^{2}$, Z. ĎURAČKOVÁ ${ }^{2}$, J. BREZA $^{\mathbf{1}}$, J. MUCHOVÁ \\ *These authors contributed equally to this work.
}

${ }^{1}$ Department of Urology, Faculty of Medicine, Comenius University and University Hospital Bratislava, Slovakia, ${ }^{2}$ Institute of Medical Chemistry, Biochemistry and Clinical Biochemistry, Faculty of Medicine, Comenius University, Bratislava, Slovakia

Received January 20, 2019

Accepted May 2, 2019

Epub Ahead of Print June 6, 2019

\begin{abstract}
Summary
Erectile dysfunction (ED) and diabetes mellitus (DM) share common pathophysiological risk factors including endothelial dysfunction which together with hyperglycemia contribute to the increased oxidative/glycooxidative stress. A reduced NO concentration is insufficient for relaxation processes in the penis. Chronic inflammation and endoglin are involved in the regulation of endothelial function. Adiponectin from the adipose tissue has anti-inflammatory effects. Our study aimed to investigate the relation between erectile function in patients with and without DM and the oxidative stress, hormone adiponectin, and endothelial dysfunction marker endoglin. Men $(n=32)$ with ED evaluated by the International Index of Erectile function (IIEF-5) questionnaire (17 without DM (NDM); 15 with type 2 diabetes mellitus (DM)) and 31 controls were included. Advanced glycation end products (AGEs), 8-isoprostanes (8-isoP), protein carbonyls, antioxidant capacity, adiponectin and endoglin were determined in the blood. DM patients compared to NDM patients and controls, had increased levels of glucose, C-reactive protein, triacylglycerols, 8-isoP, AGEs, endoglin and BMI. IIEF-5 score, NO and adiponectin levels were decreased. We are the first to find out that endoglin shows a negative correlation with erectile function in NDM, but not in DM patients. Endoglin can be considered as endothelial dysfunction marker in nondiabetic men suffering from ED.
\end{abstract}

\section{Key words}

Adiponectin • Diabetes mellitus • Endoglin • Erectile dysfunction - Oxidative stress

\section{Corresponding author}

J. Muchová, Institute of Medical Chemistry, Biochemistry and
Clinical Biochemistry, Faculty of Medicine, Comenius University, Sasinkova 2, 81372 Bratislava, Slovakia. E-mail: jana.muchova@fmed.uniba.sk

\section{Introduction}

Erectile dysfunction (ED) is characterized as the inability to achieve or maintain an erection to complete sexual intercourse or another chosen sexual activity sufficiently (Kamenov 2015, Laumann and Waite 2008). It is known that ED is more widespread in patients with type 2 diabetes mellitus and metabolic syndrome (Esposito et al. 2005).

In a pathophysiological cascade responsible for endothelial dysfunction plays a role an impaired vascular nitric oxide (NO) synthesis and impaired vasodilatation through increased inflammation and oxidative stress (Derosa et al. 2015).

ED and cardiovascular diseases have the same risk factors as hypertension, diabetes mellitus, hypercholesterolemia and smoking (Greenstein et al. 1997, Feldman et al. 1994). The severity of erectile dysfunction correlates with the cardiovascular risk of patients and represents the early symptom of generalized atherosclerosis (Baumhäkel et al. 2009). ED can also be considered as a marker of early subclinical coronary artery damage and thus as an independent predictor of future cardiovascular events (Montorsi et al. 2009).

Although ED is present in many non-diabetic men, ED may be considered as an early diabetic 
complication (Jakuš and Rietbrock 2004, Jakuš et al. 2014). However, in the case of diabetes mellitus (DM) some diabetes specific aspects should be taken into account: pathogenesis of diabetic ED is specific and more complex compared to non-diabetic men; ED is more severe and significantly affects patient lives and the effectiveness of the treatment is lower in diabetic than in non-diabetic men (Kamenov 2015).

People with DM have an increased risk of developing a number of health problems resulting in the lower life quality, including ED. Pathophysiology of DM and ED has some common risk factors including endothelial dysfunction, metabolic syndrome, insulin resistance and dyslipidemia. Combined with hyperglycemia, they participate in increased production of pro-inflammatory factors, increased oxidative stress and visceral adiposity which lead to vasoconstriction pathways and decreased NO availability in corpora cavernosa resulting in ED (Maiorino et al. 2018).

NO bioavailability may be decreased by suppressed endothelial nitric oxide synthase (eNOS) expression and/or activity or by increased NO scavenging, for example, by superoxide formed during increased oxidative stress (Ďuračková 2014).

Oxidative stress is a key pathogenic factor in the development of diabetic complications. During chronic hyperglycemia, advanced glycation end products (AGEs) are formed. AGEs bind covalently to the vascular collagen leading to thickening of the vascular wall, decreased elasticity, endothelial dysfunction and atherosclerosis (Bucala et al. 1991). During this process reactive oxygen species (ROS) are formed. Produced superoxide can react with $\mathrm{NO}$ to form peroxynitrite $\left(\mathrm{ONOO}^{-}\right)$thus reducing the concentration of NO needed for the relaxation processes and, at the same time, formed peroxynitrite contributes to the oxidative damage to significant biomolecules (Ďuračková 2010, Ďuračková 2014). ROS are involved in many mechanisms of initiation or maintenance of functional and structural damage to cells and the entire organism. Increased oxidative stress and expression of inflammatory markers are seen in patients with diabetic ED (Castela et al. 2015).

Endoglin, which is a $180 \mathrm{kDa}$ homodimeric integral membrane glycoprotein, is involved in the regulation of endothelial function, serving as a receptor for the transforming growth factor-beta superfamily involved in the atherosclerotic processes (Emeksiz et al. 2016). A soluble form of endoglin (S-endoglin), formed by the cleavage of the extracellular domain of the entire endoglin molecule, has been also suggested as a marker of endothelial dysfunction. S-endoglin was found to be increased in the serum of patients with type $2 \mathrm{DM}$ and positively correlated with the severity of diabetic vascular changes (Blázquez-Medela et al. 2010).

ED is often related to metabolic disorders associated with chronic low-grade inflammation, which has been indicated as a potential mediator of endothelial dysfunction. Visceral adiposity is thought to be the starting condition of the inflammatory state through the release of inflammatory cytokines, adhesion molecules, chemokines and through the inhibition of antiatherogenic factor, adiponectin. Adiponectin is a protein hormone secreted from the adipose tissue into the blood, which modulates a number of metabolic processes, including glucose and fatty acids oxidation (Maiorino et al. 2018). Adiponectin has anti-inflammatory effects contributing to its protective role against metabolic stress in obesity (Cao 2014, Housa et al. 2006). Adiponectin levels are decreased in type 2 diabetic patients ( $\mathrm{Li}$ et al. 2009). Recent studies have demonstrated that adiponectin has a vasoprotective role and increases the bioavailability of nitric oxide (Tsuda et al. 2011). Reduced levels of adiponectin have been observed in patients suffering from ED and were dependent on the severity of metabolic status characterized by overweight (Cao 2014).

The aim of our study was to investigate the relation between ED in patients with and without DM and the oxidative stress, anti-inflammatory hormone adiponectin and endothelial dysfunction marker, endoglin.

\section{Methods and Subjects}

\section{Ethics committee approval}

The project was approved by the Ethics committee of the Faculty of Medicine, Comenius University and the University Hospital, Bratislava, Slovakia (May 6, 2003) and was conducted in compliance with the guidelines laid down in the Declaration of Helsinki 1964. Written informed consent was obtained from all subjects.

\section{Subjects}

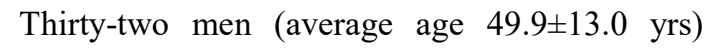
suffering from ED and registered at the Department of Urology, Faculty of Medicine, Comenius University and the University Hospital, Bratislava, Slovakia were 
included in our study. In the patient group, 17 patients were without diabetes mellitus (NDM) and 15 patients with type 2 diabetes mellitus (DM). The control group without ED consisted of 21 volunteers.

\section{Clinical investigation}

Patients with acute inflammatory diseases, renal failure, hepatic insufficiency, endocrine abnormalities (testosterone deficit) and psychiatric disorders were excluded from the study. No patient with a clear psychogenic ED was included. All patients enrolled in the study had one of the ED risk factors and were treated with phosphodiesterase type 5 inhibitors with a good response (Table 1).

Erectile function was evaluated by the International Index of Erectile Function (IIEF-5) questionnaire. Patients and volunteers filled out the IIEF-5 questionnaire during their appointment at the Urology department.

The maximum score of IIEF-5 questionnaire is 25 points (Rhoden et al. 2002). Erectile function is characterized by the following score: normal function (21-25 points), mild erectile dysfunction (16-20 points), moderate erectile dysfunction (11-15 points), severe dysfunction (less than 10 points).

\section{Biochemical parameters}

Plasma was obtained by centrifugation (10 min at $1200 \mathrm{xg}$ ) of blood using EDTA as an anticoagulant agent. Serum was obtained by the standard procedure from blood samples by centrifugation in the absence of anticoagulant. Blood plasma and serum aliquoted samples were kept at $-80^{\circ} \mathrm{C}$ until analyses.

Basic biochemical parameters glucose, triacylglycerols (TAG), high sensitive C-reactive protein (hsCRP) in serum were determined at the Department of Laboratory Medicine, University Hospital by standard biochemical procedures using the Hitachi 911 automatic analyser (Roche, Switzerland).

The antioxidant capacity of plasma was determined by TEAC method (trolox equivalent antioxidant capacity) on the Hitachi 911 automated analyser using the Randox set (United Kingdom). Antioxidant activity was expressed in $\mathrm{nmol}$ trolox $/ \mathrm{ml}$ of plasma (Re et al. 1999).

Glycated hemoglobin (HbAlc) was determined by HPLC (DiaStat, Bio-RAD, USA) in fair capillary blood using the International Federation of Clinical Chemistry (IFCC) calibration.
Advanced Glycation End products (AGEs) - the levels of advanced glycation end products (AGEs) were measured as the total fluorescence (PERKIN-ELMER LS45) of plasma at excitation/emission wavelengths $345 / 465 \mathrm{~nm}$ and expressed in arbitrary units per gram of proteins (AU/g prot.) (Kalousová et al. 2002).

Protein carbonyls (nmol/mg prot.) in blood plasma were determined using ELISA according to Buss et al. (1997) and modified by Sitte et al. (1998).

8-isoprostanes (8-isoP) in plasma $(\mathrm{pg} / \mathrm{ml})$ were determined by the 8-isoprostane EIA kit (Cayman, No. 516351).

Nitroxide - the total nitric oxide was determined in plasma as total nitrite $\left(\mathrm{NO}_{2}^{-}\right)(\mu \mathrm{mol} / \mathrm{l})$ by $\mathrm{R} \& \mathrm{D}$ Systems, Germany, DE1600 kit.

Adiponectin $(\mu \mathrm{g} / \mathrm{ml})$ was determined in serum by ELISA (BioVendor Human Adiponectin, $\breve{C} R$ ).

Endoglin $(\mathrm{ng} / \mathrm{ml})$ was determined in serum by the EIA kit (R \& D Systems Quantikine Human Endoglin/CD105 Immunoassay, USA).

\section{Statistics}

Data are presented as mean $\pm \mathrm{SD}$ or as median (centile 5-95) depending on the normality of data.

Differences in measured parameters between patient groups and controls were evaluated by the Student t-test or the Mann-Whitney $U$ test depending on the normality of data.

Correlations were evaluated by Pearson's or Spearman's tests, depending on the normality of the data. Statistical analyses were performed with StatsDirect ${ }^{\circledR}$ 3.1.22 (StatsDirect Sales, Sale, Cheshire, M33 3UY, UK) and IBM SPSS Statistics 23. $\mathrm{P}<0.05$ was considered as significant in all statistical analyses.

\section{Results}

\section{Baseline characteristics}

Patient with ED and healthy volunteer characteristic biometrical parameters and andrology data such as age, clinical variables (e.g. erectile dysfunction score IIEF, time since ED diagnosis, treatment of DM and ED, glucose, triacylglycerol levels, BMI) are summarized in the table 1 to characterize the study population and to compare baselines of investigated groups.

\section{Clinical parameters}

The median value of IIEF-5 in NDM group was 
at the upper limit of the range of severe ED and was significantly different from the IIEF-5 of the DM group, which was in the range of severe ED $(p=0.023)$. Both groups of patients (DM and NMD) were significantly different from controls $(\mathrm{p}<0.0001)$ (Table 1$)$.
Investigated group of DM patients was significantly different in BMI compared to the NDM group ( $p=0.004)$ as well as to controls $(p=0.028)$. BMI in the NDM group was not significantly different from the controls (Table 1).

Table 1. Clinical characteristics and andrology data of patients suffering from erectile dysfunction

\begin{tabular}{|c|c|c|c|}
\hline \multirow[b]{2}{*}{ Parameter } & \multicolumn{2}{|c|}{ ED } & \multirow[b]{2}{*}{$\mathbf{C}$} \\
\hline & NDM & DM & \\
\hline$n$ & 17 & 15 & 21 \\
\hline Age (years) & $49.0 \pm 11$ & $48.9 \pm 15$ & $49.6 \pm 4.4$ \\
\hline Smokers & 10 & 7 & 0 \\
\hline Arterial hypertension & 7 & 8 & 0 \\
\hline Ischemic heart disease & 3 & 4 & 0 \\
\hline Stress & 5 & 7 & 0 \\
\hline Time since ED diagnosis (months) & $12(3-48)$ & $27(4-57)^{*}$ & - \\
\hline DM treatment $-P A D$ & - & 11 & - \\
\hline - insulin & - & 2 & - \\
\hline $\begin{array}{c}\text { - antidiabetic } \\
\text { diet }\end{array}$ & - & 2 & - \\
\hline IIEF-5 in median (Centile 5-95) & $11(8-20)^{\#}$ & $10(5-12)^{* \#}$ & $23(22-25)$ \\
\hline severe ED (IIEF-5, 0-10) (\%) & $8(47)$ & $8(53)$ & - \\
\hline moderate ED (IIEF-5, 11-15) (\%) & $6(35)$ & $5(34)$ & - \\
\hline mild ED (IIEF-5, 16-20) (\%) & $3(18)$ & $2(13)$ & - \\
\hline no ED (IIEF -5, 21-25) (\%) & - & - & 21 \\
\hline ED treatment - viagra & 2 & 2 & - \\
\hline - cialis & 2 & 2 & - \\
\hline - alprostadil inj. & - & 1 & - \\
\hline$B M I\left(k g / m^{2}\right)$ & $28.3 \pm 2.9$ & $31.9 \pm 1.0 *^{\#}$ & $28.2 \pm 5.4$ \\
\hline Glucose (mmol/l) & $5.24 \pm 0.34$ & $11.06 \pm 2.3 *^{\#}$ & $5.28 \pm 0.48$ \\
\hline $\operatorname{HbAlc}(\%)$ & $3.96 \pm 0.34$ & $7.58 \pm 2.05^{* \#}$ & $3.80 \pm 0.42$ \\
\hline$h s C R P(m g / l)$ & $4.16 \pm 0.67^{\#}$ & $4.70 \pm 0.70 * \#$ & $1.94 \pm 0.98$ \\
\hline$T A G(\mathrm{mmol} / \mathrm{l})$ & $1.46 \pm 0.64$ & $2.49 \pm 1.30 *$ & $1.90 \pm 0.50$ \\
\hline
\end{tabular}

ED - erectile dysfunction, NDM - patients with erectile dysfunction and without diabetes, DM - patients with erectile dysfunction and with diabetes mellitus, IIEF- 5 - International index of erectile function, $\mathrm{C}$ - control group, n- number of patients, hsCRP - high sensitive C-reactive protein, HbA1c - glycated hemoglobin, TAG - triacylglycerols, BMI - body mass index, PAD - peroral antidiabetics *NDM vs. DM, ${ }^{\#}$ NDM or DM vs. C

\section{Biochemical parameters}

Patients with DM had significantly increased glucose levels compared to patients without DM $(\mathrm{p}<0.001)$ and to controls $(\mathrm{p}<0.001)$, while NDM did not differ from the controls.

The CRP levels in the DM and in the NDM groups were higher than in the controls (both $\mathrm{p}<0.001$ ), as well as in the DM was higher than in the NDM group $(p=0.032)$.
Higher level of TAG was observed in ED patients with DM compared to those without DM $(p=0.014)$. TAG levels in NDM and DM groups were not significantly different from the controls (Table 1).

AGEs level in the DM group were, as expected, increased compared to NDM $(\mathrm{p}=0.004)$ as well as to the controls $(p<0.001)$, while AGEs level in NDM group was not significantly different from the controls (Table 2). 
Table 2. The levels of AGEs, hormone adiponectin, metabolite endoglin and markers of oxidative stress

ED

\begin{tabular}{lccc} 
Parameter & NDM & DM & C \\
\hline$n$ & 17 & 15 & 21 \\
AGEs $($ AU/g prot. $)$ & $153.8 \pm 40.6$ & $277.0 \pm 89.4^{* \#}$ & $136 \pm 28$ \\
Endoglin $(\mathrm{ng} / \mathrm{ml})$ & $3.85 \pm 0.6$ & $4.53 \pm 0.55^{* \#}$ & $3.48 \pm 0.68$ \\
Adiponectin $(\mu \mathrm{g} / \mathrm{ml})$ & $1.80 \pm 0.33$ & $1.34 \pm 0.4^{*}$ & $1.78 \pm 0.8$ \\
8-isoP $(\mathrm{pg} / \mathrm{ml})$ & $44.57 \pm 12.5^{\#}$ & $67.3 \pm 15.1^{* \#}$ & $21.69 \pm 10.4$ \\
Carbonyls of proteins & $0.45 \pm 0.06$ & $0.46 \pm 0.08$ & $0.48 \pm 0.04$ \\
(nmol/mg) & $0.225 \pm 0.04^{\#}$ & $0.226 \pm 0.03^{\#}$ & $1.07 \pm 0.34$ \\
TEAC $(\mathrm{mmol} / \mathrm{l})$ & $44.32 \pm 8.8^{\#}$ & $25.03 \pm 6.8^{* \#}$ & $55.5 \pm 9.1$ \\
NO $(\mu \mathrm{mol} / \mathrm{l})$ & & &
\end{tabular}

ED - erectile dysfunction, NDM - patients with erectile dysfunction and without diabetes, DM - patients with erectile dysfunction and with diabetes mellitus, $\mathrm{C}$ - control group, $\mathrm{n}$ - number of patients, AGEs - advanced glycation end products, 8-isoP - 8-isoprostanes, TEAC - trolox equivalent antioxidant capacity. * NDM vs. DM, ${ }^{*} \mathrm{NDM}$ or DM vs. C

The level of endoglin was significantly increased in the DM group compared to the NDM group $(\mathrm{p}=0.004)$ and to controls $(p<0.001)$. Endoglin levels in the NDM group were marginally increased compared to the controls $(\mathrm{p}=0.082)$ (Table 2).

Adiponectin in NDM group was not significantly different from the controls and in the DM group it was marginally decreased compared to the controls $(p=0.067)$. However, the difference between NDM and DM groups was significant $(p<0.001)$ (Table 2).

8-isoP were significantly increased in the DM and NDM groups compared to the controls $(\mathrm{p}<0.001)$. Moreover, DM group had 8-isoP significantly higher than NDM group $(p=0.003)$. Protein carbonyls were not different in DM, NDM groups and in controls.

Antioxidant capacity was not different between DM and NDM groups, but in both groups, it was lower than in the controls $(\mathrm{p}<0.001)$.

The NO level was lower in both DM $(p=0.003)$ and NDM $(p=0.028)$ groups compared to controls. The level of NO in the DM group, was decreased compared to NDM group $(\mathrm{p}<0.001)$ (Table 2$)$.

As expected, significant positive correlation has been found between glucose and AGEs in the DM group, but not in the NDM. Increased AGEs in the DM group, but not in the NDM group, correlated with increased endoglin. This association was similar to the association between glucose and endoglin in the DM group, but not in the NDM group (Table 3). Endoglin in the NDM group showed a negative correlation with NO level and with
IIEF-5, while in the DM group these correlations were not significant. In addition, we have confirmed a negative correlation between NO level and IIEF score only in the NDM group, but not in the DM group (Table 3).

Adiponectin showed the expected association with TAG in the DM group and marginal association in the NDM group $(\mathrm{r}=-0.400, \mathrm{p}=0.055)$ (Table 3$)$. However, 8-isoP, protein carbonyls, NO, IIEF-5 and glucose levels did not show any associations with adiponectin in both, DM and NDM groups. IIEF score negatively correlated with high-sensitive C-reactive protein (hsCRP) in both groups (Table 3).

Antioxidant capacity of plasma determined as TEAC was not associated either with the glucose level or IIEF score in the DM group. However, in the NDM group TEAC showed a correlation with the IIEF score (Table 3).

Time since ED diagnosis was not associated either with endoglin and adiponectin or with IIEF-5 in both groups (DM and NDM).

\section{Discussion}

Two groups of patients with erectile dysfunction (ED), with and without DM comorbidity have been examined for oxidative stress markers, the marker of adipose tissue - adiponectin and the marker of endothelial cell activity - endoglin. Patients with ED and diabetes had worse symptoms of erectile function, increased oxidative stress, endothelial activity and reduced antioxidant ability and adiponectin compared to ED patients without DM 
and to the controls. Alterations in the NO/cGMP pathways and other vasculogenic, neurogenic, hormonal, veno-occlusive and/or pharmacogenic factors including oxidative stress contribute to the pathophysiological mechanisms of ED (Melman and Rehman 1999).

Table 3. Correlations between variables

\begin{tabular}{lcc}
\hline \multicolumn{1}{c}{ Correlations } & DM group & NDM group \\
\hline glucose vs. $A G E s$ & $\mathrm{r}=0.598, \mathrm{p}=0.016$ & n.s. \\
AGEs vs. endoglin & $\mathrm{r}=0.648, \mathrm{p}=0.018$ & n.s. \\
glucose vs. endoglin & $\mathrm{r}=0.663, \mathrm{p}=0.007$ & n.s. \\
endoglin vs. $N O$ & n.s. & $\mathrm{r}=-0.628, \mathrm{p}=0.008$ \\
endoglin $v$ s. $I I E F-5$ & n.s. & $\mathrm{r}=-0.566, \mathrm{p}=0.009$ \\
NO vs. IIEF-5 & n.s. & $\mathrm{r}=-0.626, \mathrm{p}=0.008$ \\
adiponectin $v s$. TAG & $\mathrm{r}=-0.515, \mathrm{p}=0.032$ & $\mathrm{n} . \mathrm{s}$. \\
IIEF-5 vs. $C R P$ & $\mathrm{r}=-0.888, \mathrm{p}<0.001$ & $\mathrm{r}=-0.764, \mathrm{p}<0.001$ \\
TEAC vs. $I I E F-5$ & n.s. & $\mathrm{r}=0.857, \mathrm{p}<0.001$ \\
\hline
\end{tabular}

AGEs - advanced glycation end products, CRP - C-reactive protein, DM - patients with erectile dysfunction and with diabetes mellitus, IIEF-5 - international index of erectile function, NDM - patients with erectile dysfunction and without diabetes, TAG - triacylglycerols, TEAC - trolox equivalent antioxidant capacity, n.s. - non-significant

Our results confirmed that diabetes mellitus significantly contributes to the pathophysiology and manifestation of ED. ED is present in many non-diabetic men (NDM), but in diabetics, ED may appear before diabetes is diagnosed, as both diagnoses combine several common features, including endothelial dysfunction (Kamenov 2015).

Longer duration of ED in DM patients than in patients without DM (Table 1) is consistent with the Kamenov review (2015). Diabetes has been associated with higher levels of blood glucose, hsCRP and BMI compared to NDM patients and to healthy controls (Table 1). TAG levels were higher in DM compared to NDM, but not to the controls. A similar association of ED in Italian DM patients was found by Derosa et al. (2015). Long-term type 2 diabetes leads to the development of advanced glycation end products (AGEs), which have also been confirmed in our patients with DM. Patients in DM group, but not in NDM group, had increased AGEs by $103 \%$ compared to controls.

Diabetes is one of the leading causes of cardiovascular disease associated with number of health problems lowering quality of life, including sexual dysfunction. Diabetes and ED share several cardiometabolic risk factors, such as endothelial dysfunction, obesity, metabolic syndrome and hormonal inadequacy (Melman and Rehman 1999). These factors, together with hyperglycemia, participate in endothelial activation, pro-inflammatory state and oxidative stress (Maiorino et al. 2014). Increased vasoconstriction and reduced NO availability in corpora cavernosa results in limited erectile function (Maiorino et al. 2018).

To investigate the relationship between endothelial and erectile functions in diabetics and nondiabetics, we evaluated the level of circulating soluble endoglin produced by activated endothelial cells. We have found increased level of endoglin in patients with ED without DM (NDM) compared to controls, and in ED patients with DM compared to the NDM patients. According to our information (PubMed) there has been no work published on the levels of endoglin in patients with ED.

In 64 patients with type 2 diabetes a borderline positive correlation between endoglin and blood glucose $(\mathrm{r}=0.221, \mathrm{p}=0.079)$ was observed by Blázquez-Medela et al. (2010). Emeksiz et al. (2016) found increased level of endoglin in adolescents with type 1 DM. Authors concluded that endoglin concentration might be increased in parallel with the deterioration in endothelial function before subclinical structural vascular alterations become evident. We have found a positive correlation between basal glycemia or AGEs and endoglin in DM, but not in NDM patients (Table 3). It confirms that diabetes contributes to the endothelial dysfunction.

Negative correlations between endoglin and NO as well as between endoglin and IIEF-5 found in NDM 
group confirm the relationship of ED with endothelial dysfunction. However, in the DM group, these correlations were not significant, suggesting that DM contributes to the pathophysiology of ED with other features as well. The positive correlation between NO and IIEF-5 in the NDM, but not in DM groups confirmed the negative impact of diabetic conditions including endothelial dysfunction on erectile function.

Erectile dysfunction is a frequent comorbidity of obesity (Han et al. 2011). Our group of DM, but not NDM patients had increased BMI compared to the controls. Similarly, TAG levels were increased in DM compared to NDM patients. For the obesity is characteristic a pro-inflammatory state (Wang et al. 2013) which represents an increased risk for cardiovascular diseases, endothelial dysfunction, and sexual dysfunction (Maiorino et al. 2018).

Esposito et al. (2005) found higher concentration of CRP in men with metabolic syndrome compared to controls. Our ED patients had increased CRP in DM and NDM groups compared to controls.

Obesity, metabolic syndrome, and type 2 diabetes are associated with the under-expression of adiponectin which exhibits anti-inflammatory (Cao 2014), anti-atherogenic properties (Li et al. 2009) and vascular-protective activities by suppressing endothelial cell apoptosis (Kobayashi et al. 2004).

We have observed a negative correlation between adiponectin and TAG in the DM group, but no correlation between adiponectin and BMI. Adiponectin showed no correlations with any markers of erectile function (NO, IIEF-5), diabetes (glucose, AGEs), inflammation (CRP) or oxidative stress markers (8-isoP, protein carbonyls) in both groups, which is inconsistent with Ghoshal and Bhattacharyya (2015).

Erectile dysfunction is a prevalent complication of diabetes, and oxidative stress is an important feature of diabetic ED. Oxidative stress-induced damage plays a pivotal role in the development of tissue alterations (Castela et al. 2015). Authors' findings from animal experiments with rats indicate that the deleterious effects of oxidative stress are more prominent in late diabetes. Increased penile protein oxidative modifications and decreased eNOS expression may be responsible for structural and/or functional deregulation, contributing to the progression of diabetes-associated ED. Musicki et al. (2017) studied effects of uncoupling eNOS and nNOS in penile erectile tissue on ED pathogenesis in patients compared to healthy individuals and concluded that also uncoupled eNOS and nNOS can contribute to ED pathology. We have not found a difference in the systemic marker of oxidative damage to proteins protein carbonyls in both groups of patients (NDM and DM). However, the situation in the penile tissue may be different from that found in the blood, which supports the results of Castela et al. (2015). Conversely, the marker of oxidative damage to lipids, 8-isoprostanes, were increased in both groups of ED patients compared to the controls (205\% and $310 \%$, resp.). Total antioxidant capacity of plasma was reduced in both groups by around $80 \%$. However, the correlation between antioxidant capacity (determined as TEAC) and IIEF-5 score was not observed in diabetics, while NDM patients showed a strong positive association. Markers of oxidative stress (protein carbonyls, 8-isoprostanes, antioxidant capacity) did not correlate with any other ED markers under investigation.

We realize that a certain limitation of our work is the unequal representation of smokers in the control and patient groups. Controversial results were found on the effect of smoking on erectile dysfunction (Jandíková et al. 2017). In our set of patients, no significant difference in either IIEF-5 score or biochemical parameters between smokers and non-smokers was observed.

\section{Conclusions}

We are the first who have found that endothelial cell activity marker, endoglin showed a negative correlation with erectile function in NDM patients, but not in DM patients. Endoglin can be considered as the endothelial dysfunction marker in men suffering from erectile dysfunction.

\section{Conflict of Interest}

There is no conflict of interest.

\section{Acknowledgements}

Authors wish to thank L'. Chandogová and D. Opálená for their technical assistance and L. Míková for help with references. We also thank Paul Henning for the English language editing. This study was supported by the VEGA grants 1/4306/07, 1/4309/07 of Ministry of Education of the Slovak Republic. 


\section{References}

BAUMHÄKEL M, SCHLIMMER N, KRATZ MT, BÖHM M: Erektile dysfunktion: indikator für endorganschädigung beim kardiovaskulären patienten (In German). Med Klin 104: 309-313, 2009.

BLÁZQUEZ-MEDELA AM, GARCÍA-ORTIZ L, GÓMEZ-MARCOS MA, RECIO-RODRÍGUEZ JI, SÁNCHEZRODRÍGUEZ A, LÓPEZ-NOVOA JM, MARTÍNEZ-SALGADO C: Increased plasma soluble endoglin levels as an indicator of cardiovascular alteration ns in hypertensive and diabetic patients. BMC Med 8: 86, 2010.

BUCALA R, TRACEY KJ, CERAMI A: Advanced glycosylation products quench nitric oxide and mediate defective endothelium-dependent vasodilatation in experimental diabetes. J Clin Invest 87: 432-438, 1991.

BUSS H, CHAN TP, SLUIS KB, DOMINGAN NM, WINTERBOURN CC: Protein carbonyl measurement by a sensitive ELISA method. Free Radic Biol Med 23: 361-366, 1997.

CAO H: Adipocytokines in obesity and metabolic disease. J Endocrinol 220: T47-T59, 2014.

CASTELA A, GOMES P, DOMINGUES VF, PAÍGA P, COSTA R, VENDEIRA P, COSTA C: Role of oxidative stress-induced systemic and cavernosal molecular alterations in the progression of diabetic erectile dysfunction. J Diabetes 7: 393-401, 2015.

DEROSA G, ROMANO D, TINELLI C, D'ANGELO A, MAFFIOLI P: Prevalence and associations of erectile dysfunction in a sample of Italian males with type 2 diabetes. Diabetes Res Clin Pract 108: 329-335, 2015.

ĎURAČKOVÁ Z: Free radicals and antioxidants for non-experts. In: Systems Biology of Free Radicals and Antioxidants. I LAHER (ed). Springer-Verlag Berlin Heidelberg, 2014, pp. 3-38.

ĎURAČKOVÁ Z: Some current insights into oxidative stress. Physiol Res 59: 459-469, 2010.

EMEKSIZ HC, BIDECI A, DAMAR Ç, DERINKUYU B, ÇELIK N, DÖĞER E, YÜCE Ö, ÖZMEN MC, ÇAMURDAN MO, CINAZ P: Soluble endoglin level increase occurs prior to development of subclinical structural vascular alterations in diabetic adolescents. J Clin Res Pediatr Endocrinol 8: 313-320, 2016.

ESPOSITO K, GIUGLIANO F, MARTEDİ E, FEOLA G, MARFELLA R, D'ARMIENTO M, GIUGLIANO D: High proportions of erectile dysfunction in men with the metabolic syndrome. Diabetes Care 28: 1201-1203, 2005.

FELDMAN HA, GOLDSTEIN I, HATZICHRISTOU DG, KRANE RJ, MCKINLAY JB: Impotence and its medical and psychosocial correlates: results of the Massachusetts male aging study. J Urol 151: 54-61, 1994.

GHOSHAL K, BHATTACHARYYA M: Adiponectin. Probe of the molecular paradigm associating diabetes and obesity. World J Diabetes 15: 151-166, 2015.

GREENSTEIN A, CHEN J, MILLER H, MATZKIN H, VILLA Y, BRAF Z: Does severity of ischemic coronary disease correlate with erectile function? J Impot Res 9: 123-126, 1997.

HAN TS, TAJAR A, O'NEILL TW, JIANG M, BARTFAI G, BOONEN S, CASANUEVA F, FINN JD, FORTI G, GIWERCMAN A, HUHTANIEMI IT, KULA K, PENDLETON N, PUNAB M, SILMAN AJ, VANDERSCHUEREN D, LEAN ME, WU FC; EMAS GROUP: Impaired quality of life and sexual function in overweight and obese men: the european male ageing study. Eur Endocrinol 164: 1003-1011, 2011.

HOUSA D, HOUSOVÁ J, VERNEROVÁ Z, HALUZÍK M. Adipocytokines and cancer. Physiol Res 55: 233-244, 2006.

JAKUŠ V, RIETBROCK N: Advanced glycation end-products and the progress of diabetic vascular complications. Physiol Res 53: 131-142, 2004.

JAKUŠ V, SÁNDOROVÁ E, KALNINOVÁ J, KRAHULEC B: Monitoring of glycation, oxidative stress and inflammation in relation to the occurrence of vascular complications in patients with type 2 diabetes mellitus. Physiol Res 63: 297-309, 2014.

JANDÍKOVÁ H, DUŚKOVÁ M, STÁRKA L: The influence of smoking and cessation on the human reproductive hormonal balance. Physiol Res 66: S323-S331, 2017.

KALOUSOVÁ M, SKRHA J, ZIMA T: Advanced glycation end-products and advanced oxidation protein products in patients with diabetes mellitus. Physiol Res 51: 597-604, 2002.

KAMENOV ZA: A comprehensive review of erectile dysfunction in men with diabetes. Exp Clin Endocrinol Diabetes 123: 141-158, 2015. 
KOBAYASHI H, OUCHI N, KIHARA S, WALSH K, KUMADA M, ABE Y, FUNAHASHI T, MATSUZAWA Y: Selective suppression of endothelial cell apoptosis by the high molecular weight form of adiponectin. Circ Res 94: 27-31, 2004.

LAUMANN EO, WAITE LJ: Sexual dysfunction among older adults: prevalence and risk factors from a nationally representative U.S. probability sample of men and women 57-85 years of age. J Sex Med 5: 2300-2311, 2008.

LI S, SHIN HJ, DING EL, VAN DAM RM: Adiponectin levels and risk of type 2 diabetes: a systematic review and meta-analysis. JAMA 302: 179-188, 2009.

MAIORINO MI, BELLASTELLA G, ESPOSITO K: Diabetes and sexual dysfunction: current perspectives. Diabetes Metab Syndr Obes 7: 95-105, 2014.

MAIORINO MI, BELLASTELLA G, GIUGLIANO D, ESPOSITO K: From inflammation to sexual dysfunctions: a journey through diabetes, obesity, and metabolic syndrome. J Endocrinol Invest 41: 1249-1258, 2018. doi: 10.1007/s40618-018-0872-6.

MELMAN A, REHMAN J: Pathophysiology of erectile dysfunction. Mol Urol 3: 87-102, 1999.

MONTORSI P, RAVAGNANI PM, GALLI S, ALI SG, BRIGANTI A, SALONIA A, MONTORSI F: The triad of endothelial dysfunction, cardiovascular disease and erectile dysfunction: Clinical Implications. European Urology Supplements 8: 58-66, 2009.

MUSICKI B, BURNETT AL: Constitutive NOS uncoupling and NADPH oxidase upregulation in the penis of type 2 diabetic me with erectile dysfunction. Andrology 5: 294-298, 2017.

RE R, PELlEGRINI N, PROTEGGENTE A, PANNALA A, YANG M, RICE-EVANS C: Antioxidant activity applying an improved ABTS radical cation decolorization assay. Free Radic Biol Med 26: 1231-1237, 1999.

RHODEN EL, TELÖKEN C, SOGARI PR, VARGAS SOUTO CA: The use of the simplified International Index of Erectile Function (IIEF-5) as a diagnostic tool to study the prevalence of erectile dysfunction. Int J Impot Res 14: 245-250, 2002.

SITTE N, MERKER K, GRUNE T: Proteasome-dependent degradation of oxidized proteins in MRC-5 fibroblasts. FEBS Lett 440: 399-402, 1998.

TSUDA K: Roles of adiponectin and oxidative stress in the regulation of membrane microviscosity of red blood cells in hypertensive men-an electron spin resonance study. J Obes Art 548140, 2011. doi: 10.1155/2011/548140.

WANG X, BAO W, LIU J, OUYANG YY, WANG D, RONG S, XIAO X, SHAN ZL, ZHANG Y, YAO P, LIU LG: Inflammatory markers and risk of type 2 diabetes: a systematic review and meta-analysis. Diabetes Care 36: 166-175, 2013. 